\title{
Rabbit Adipose-derived Stem Cells and Tibia Repair
}

\author{
Elena Arrigoni, ${ }^{a}$ Stefania Niada ${ }^{\mathrm{b}}$ and Anna T. Brini ${ }^{\mathrm{c}, *}$
}

\begin{abstract}
Adipose-derived Stem Cells (ASCs) may represent, alone or in combination with different scaffolds, a novel and efficient approach for bone regeneration. Here, we describe how autologous rabbit ASCs (rbASCs) isolated from interscapular adipose tissue, expanded and characterized in vitro, are used to regenerate a full-thickness bone defect in the tibial crest of New Zealand rabbits.

The animals have been divided in four groups: one group where the lesions have been treated with rbASCs seeded on hydroxyapatite-disk (rbASCs-HA), one group with only rbASCs, one with just HA, and one untreated group (just defect). The follow-up was of eight weeks.

Meanwhile, rbASCs have been characterized in vitro: these progenitor cells show a homogenous high proliferation rate and a marked clonogenic ability. Moreover, rbASCs demonstrate an osteogenic potential that has been evaluated by the expression of specific bone markers such as alkaline phosphatase, collagen, osteonectin and extracellular calcified matrix deposition, both in the absence and in the presence of hydroxyapatite.
\end{abstract}

Department of Biomedical, Surgical and Dental Sciences, University of Milan, Via Vanvitelli, 32, 20129 Milan-Italy. IRCCS Galeazzi Orthopaedic Institute, Via R. Galeazzi, 4, 20161 Milan-Italy.

${ }^{a}$ E-mail: elena.arrigoni@unimi.it

bE-mail: stefania.niada@unimi.it

'E-mail: anna.brini@unimi.it

*Corresponding author

List of abbreviations given at the end of the text. 
Eight weeks after surgical interventions, gross appearance, X-rays, histological analyses and biomechanical tests were performed on all the animals.

The macroscopic analyses of all the tibias show a satisfactory filling of the lesions without any significant difference in terms of stiffness. By $X$-rays, a good osteo-integration appears in both scaffold-treated groups; despite the fact that HA was not completely resorbed, cells-HA treated bones show a more efficient scaffold resorption than the other group. In addition, the scaffold-treated defects show a better bone formation compared to the control samples. In particular, the new bone, formed in the presence of rbASCs-HA, is more mature and similar to the native one showing an improvement in bone mechanical properties.

These results indicate that autologous ASCs-hydroxyapatite bioconstruct may be a potential treatment for the regeneration of bone defects.

\section{Introduction}

Until recently, fat has been considered an inert tissue and usually lipoaspirates from aesthetic surgery were discarded as surgical waste. Instead, adipose tissue is a highly complex system which consists of mature adipocytes, preadipocytes, fibroblasts, vascular smooth muscle cells, endothelial cells, resident macrophages and lymphocytes and Adiposederived Stem Cells (ASCs) (Zuk et al. 2002; de Girolamo et al. 2008). These ASCs are progenitor cells that possess the potential to differentiate into cells of mesodermal origin. Furthermore, there are evidences of the ability of ASCs to transdifferentiate into cells of non-mesodermal origin, such as neurons, endocrine pancreatic cells, hepatocytes, endothelial cells, and cardiomyocite (Schaffler and Buchler 2007).

ASCs are quite similar to mesenchymal stem cells isolated from bone marrow (BMSCs) regarding morphology, immunophenotype, colony frequency and differentiation capacity (Kern et al. 2006).

ASCs sampling is a simple surgical procedure due to the easy and repeatable access to subcutaneous adipose tissue as well as the uncomplicated enzyme-based isolation procedures. Therefore, this tissue offers a great alternative source of autologous adult stem cells that can be obtained repeatedly in large quantities under local anaesthesia, with minimum discomfort for the patient (Schaffler and Buchler 2007).

Other relevant ASCs features are their immunoregolatory properties both in vivo and in vitro and their low immunogenicity; indeed, they do not express MHC class II and express low levels of MHC class I. Both ASCs (Najar et al. 2010) and BMSCs, are able to inhibit T-cell proliferation in a dose-dependent manner (Di Nicola et al. 2002); ASCs effect appears to be 
mediated by high levels of secreted leukaemia inhibitory factor (LIF) (Najar et al. 2010).

Following these observations, in recent years the broad immunoregulatory activities of ASCs have been studied and their use in clinical therapies, such as the prevention and/or reduction of graft-versus-host disease (GVHD) and the treatment of autoimmune diseases, seems to be particularly promising (Yanez et al. 2006; Choi et al. 2012; Gonzalez et al. 2009; Constantin et al. 2009).

In parallel to these innovative and very interesting applications, ASCs have been largely studied for their regenerative ability.

Above all, to optimize tissue reconstruction, stem or stromal cells such as ASCs have been used in association with biocompatible scaffolds in the emerging field of tissue engineering. Stem cells adhere to the scaffold, replicate, differentiate and then organize into the new tissue. Generally, an ideal scaffold should be biocompatible, absorbable and highly porous. In particular, for bone regeneration, the scaffold associated with ASCs should also possess osteoconductive and osteoinductive properties and should promote cellular adherence and recruitment (Spencer et al. 2011). Currently, investigations are underway to identify scaffold properties that will optimize stem cell activity.

Although bone is a very dynamic tissue, the spontaneous regeneration is limited to relatively small defects, while large bone defects resulting from trauma, tumours, osteitis, implant loosening or corrective osteotomies require surgical treatment. Actual therapies for skeletal reconstruction include autografts or allografts, mineral bone substitutes, and callus distraction (Sarkar et al. 2005). These techniques present the risk of complications and nonunions, although with a high percentage of success. Moreover, the harvesting of autologous bone often results in donor site morbidity, whereas allografts, either from human cadaver or animals, present potential risks of infection, immune response, inadequate supply, difficulties in obtaining and processing tissue, and rapid resorption (Torroni 2009).

With this background, the possible use of ASC-scaffold constructs for treatment of bone defects constitutes a promising technique, considering also the harvesting site which allows low donor-site morbidity. In this chapter, we present an approach of tissue engineering that use autologous ASCs in a rabbit model of critical bone defect.

Rabbit is a good animal model used in approximately 35 percent of musculoskeletal research studies (Neyt et al. 1998). This is partially due to its easy handling, the use of compact caging and the relative low cost for animal purchase and care. Similar to other small animal models, the rabbit also affords the opportunity to use an adequate number of genotypically similar subjects (Chu et al. 2010). Interestingly for our research field, rabbit 
reaches skeletal maturity at around 6 mon of age, shortly after it is sexually mature (Gilsanz et al. 1988).

Concerning bone composition, some similarities between humans and rabbits are reported both in bone mineral density (BMD) and in the fracture toughness of mid-diaphyseal bone (Wang et al. 1998). One drawback in using this animal model is the difference in the bone anatomy between rabbits and humans, regarding size, shape and loading, due to the differences in weight and stance. Other differences with humans are in the rabbit bone microstructure (Wang et al. 1998) as well as the skeletal change and bone turnover, which are faster in rabbits (Castaneda et al. 2006; Gilsanz et al. 1988).

Even though these issues make extrapolation of studies performed in rabbits onto the human clinical response quite difficult, this preclinical model remains an accepted and a good tool for testing new biomedical constructs before moving towards a larger animal model.

The main sources of mesenchymal stem cells for bone regeneration studies in rabbit are bone marrow and adipose tissue. However, the type of support used is quite variable; modified or unmodified hydroxyapatite (HA) is one of the principal scaffolds employed (de Girolamo et al. 2011; Oshima et al. 2010; Li et al. 2009; Hao et al. 2010b). Other supports such as $\beta$-tricalcium phosphate (Wang et al. 2010), collagen I gel with PLGA$\beta$-TCP (Hao et al. 2010b), titanium dome (Pieri et al. 2010) and modified polycaprolactone (Im and Lee 2010) scaffolds have also been used. Even allogenic (Dudas et al. 2006) or xenogenic (Zhao et al. 2011) bone graft represent valid supports for MSCs.

Since vascularization is required to maintain cell survival, scaffold design, use of bioreactor combined with angiogenic factors and prevascularization procedure are currently under investigation for enhancing blood vessels formation in tissue engineered bone grafts (Lovett et al. 2009). Two recent studies by Zhao et al. and Wang et al. show indeed a faster capillary infiltration of the graft when a vascular bundle was previously inserted (Zhao et al. 2011; Wang et al. 2010).

Another aspect under debate is the need or not to differentiate MSCs before the construct is implanted; there are contrasting data about this issue that still needs to be elucidated (de Girolamo et al. 2011; Dudas et al. 2006; Dashtdar et al. 2011).

An interesting alternative experimental approach is to osteodifferentiate MSCs when they are already implanted into the defect. Bone Morphogenic Proteins (BMPs) are among the most potent osteoinductive factors ever discovered (Albrektsson and Johansson 2001). MSCs infected with recombinant adenovirus vector coding for human BMPs produce high levels of BMPs at target sites, obtaining a faster bone defect repair (Hao et al. 2010a; Li et al. 2010). 
Even though these strategies, to enhance osteo-differentiation of MSCs, can be useful to accelerate bone repair, however we need to keep in mind that manipulation of bioengineered constructs makes their use more difficult for future clinical application.

\section{Isolation of rabbit Adipose-derived Stem Cells (rbASCs)}

Rabbit ASCs (rbASCs) can be derived from surgically resected adipose tissue of New Zealand White Rabbits (Oryctolagus cuniculus). The source is a deposit of adipose tissue located along the dorsomedial line, approximately $5 \mathrm{~cm}$ from the skull in the craniocaudal direction (Fig. 19.1A).

The adipose tissue is easily accessible through a $2-$ to $3-\mathrm{cm}$ sagittal incision in the dorsomedial line over the tissue area (Fig. 19.1B). The amount of adipose tissue varies according to the animal mass, in a proportion of 5 to $7 \mathrm{~g}$ per kilogram of body mass: this corresponds to a mass of 20 to $30 \mathrm{~g}$ in an adult animal weighing nearly $4.5 \mathrm{~kg}$.

Once harvested, adipose specimens are finely minced and ASCs are purified with 0.1 percent collagenase type I (Dudas et al. 2006; Torres et al. 2007; Arrigoni et al. 2009). The isolated Stromal Vascular Fraction (SVF) is plated and Adipose-derived Stem Cells (ASCs) adhere to the Petri dish

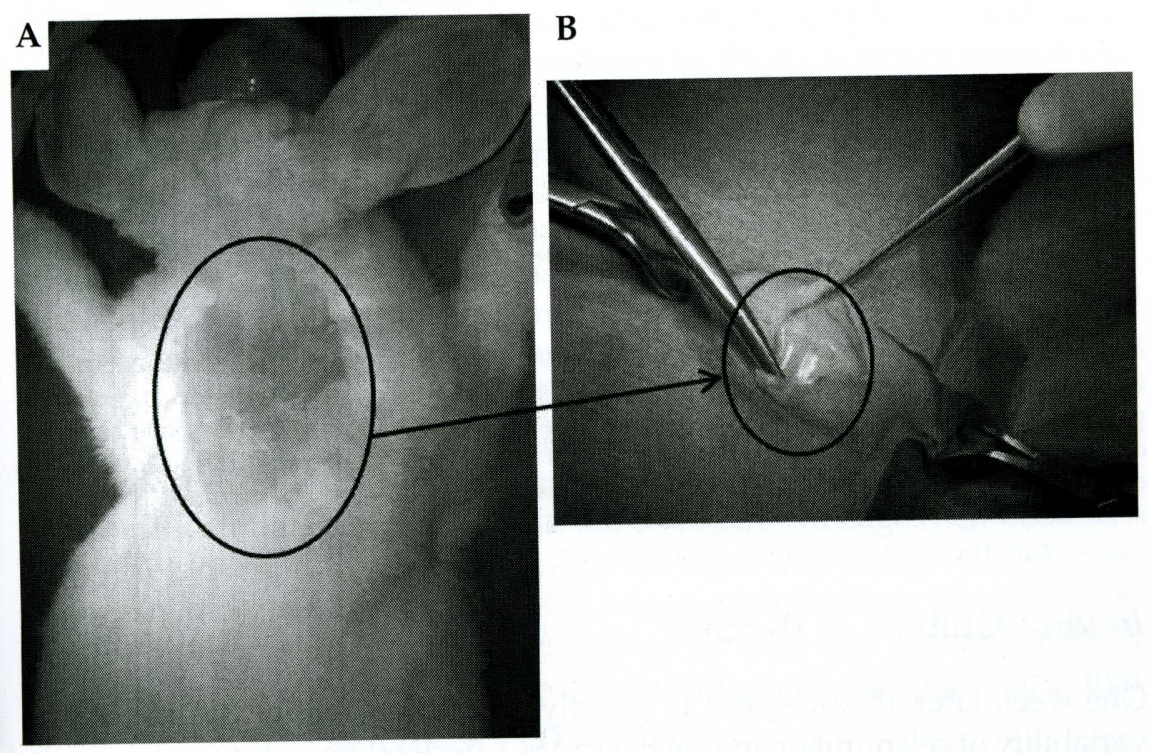

Figure 19.1 Adipose tissue localization. Rabbit dorsal region where the adipose tissue pouch is located (A). Surgical procedure for withdrawing the adipose tissue pouch (B) (unpublished data). 
(Fig. 19.2). From an average of $5.2 \pm 1.6 \mathrm{ml}$ of raw adipose tissue $2.8 \times 10^{5} \pm$ $1.9 \times 10^{5}$ cells $/ \mathrm{ml}$ are purified (Fig. 19.2E).

Rabbit ASCs are cultured at a density of $10^{5}$ cells $/ \mathrm{cm}^{2}$ in DMEM supplemented with 10 percent fetal bovine serum (FBS), $2 \mathrm{mM} \mathrm{L-glutamine,}$ $50 \mathrm{U} / \mathrm{mL}$ penicillin, and $50 \mu \mathrm{g} / \mathrm{mL}$ streptomycin (CTRL medium), and maintained at $37^{\circ} \mathrm{C}$ in a humidified atmosphere with 5 percent $\mathrm{CO}_{2}$. After 48-72 hr, non-adherent cells and erythrocytes are discarded, and, when rbASCs reach $80-90$ percent confluence, cells are detached by 0.5 percent trypsin/0.2 percent EDTA and plated at a density of $5 \times 10^{3}$ cells $/ \mathrm{cm}^{2}$ (Arrigoni et al. 2009).

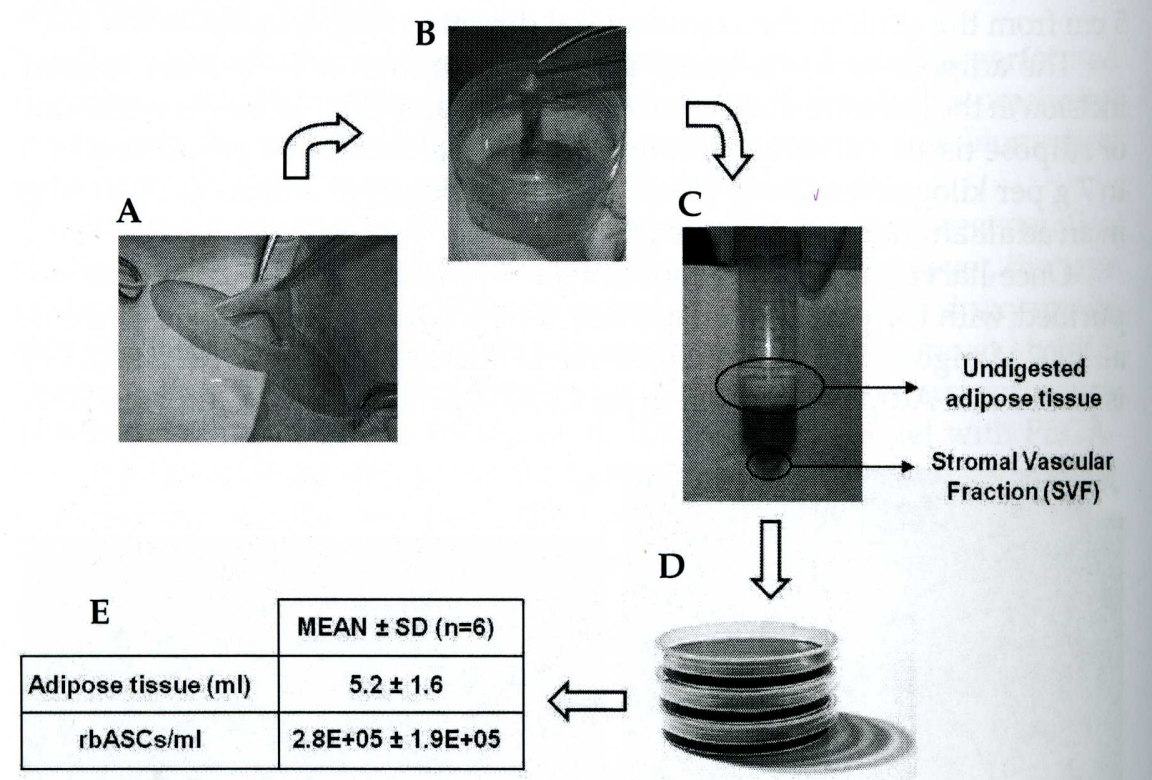

Figure 19.2 Isolation of rabbit Adipose-derived Stem Cells. Adipose tissue is harvested from interscapular region (A) and maintained in sterile PBS (phosphate-buffered saline) until processed (B). Stromal Vascular Fraction is separated by centrifugation (C), then seeded in Petri dishes and cultured in humidified atmosphere at $37^{\circ} \mathrm{C}$ with 5 percent $\mathrm{CO}_{2}$ (D) (unpublished data). Cellular yield of rbASCs per ml of adipose tissue (E) (with permission from de Girolamo et al. 2011).

\section{In vitro Culture of rbASCs}

One week later, rbASCs start to rapidly proliferate and, at passage 1 , the variability of cell number among the rbASCs derived from different animals is reduced, reaching, in $30 \mathrm{~d}, 2.6 \times 10^{8} \pm 9.9 \times 10^{7}$ cells starting from $1.5 \times 10^{5}$ rbASCs. Usually every $7 \mathrm{~d}$ these cells reach the confluence: the doubling time of $56.9 \pm 14.8 \mathrm{hr}$ is constant from passage 2 to 6 (Fig. 19.3A). These cells 

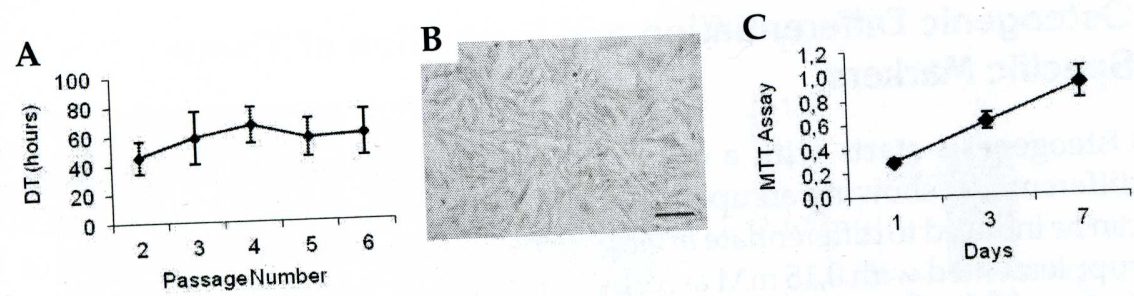

D
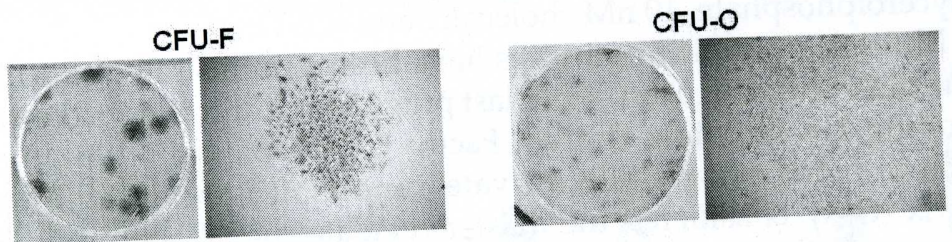

Characterization of rbASCs. Proliferation of rbASCs at early passages (A). 列 Microphotographs of fixed rbASC one week in undifferentiated condition (mean $\pm S D, n=6$ ) Viability of rbASCs maintained for one and Alizarin Red-S, respectively (D) (modified by de Girolamo et al. 2011).

show the characteristic fibroblast-like morphology (Fig. 19.3B), without any sign of cellular senescence. During one week of culture, we observed an exponential growth of rbASCs, one of the main features that characterize mesenchymal stem cells. These data are also confirmed by MTT cell viability assay (Fig. 19.3C). Cell viability is monitored by the reduction of tetrazolium salts by metabolically active cells, through the action of dehydrogenase enzymes in the mitochondria, to generate reducing agents such as NADH and NADPH.

As originally indicated by Friedenstein et al. (Friedenstein et al. 1974), another prominent feature of MSCs is their ability to generate colonies, evaluated by Colony Forming Unit (CFU) assay. rbASCs show a great clonogenic ability maintained quite constant from passage 1 to 4 , with a mean value of $3.0 \pm 1.6$ percent (mean $\pm \mathrm{SD}$ ). Moreover, these rbASCs possess the ability to produce osteoblast (CFU-O) colonies with a frequency of $4.3 \pm$ 1.9 percent (mean $\pm \mathrm{SD}$ ), highlighting the presence of osteogenic progenitors able to differentiate towards cells of the osteogenic lineage (Fig. 19.3D).

Furthermore, cryopreservation does not affect rabbit stem cells features, as already reported for human ASCs. At low temperatures any biological activity is efficiently blocked and storage at very low temperatures is presumed to provide an indefinite cellular longevity although the actual "shelf life" is rather difficult to presume. 


\section{Osteogenic Differentiation and Evaluation of Tissue Specific Markers}

Osteogenesis starts with a commitment by mesenchymal stem cells to differentiate, showing an up-regulation of osteoblast-specific genes. ASCs can be induced to differentiate in vitro, by maintaining them in CTRL medium supplemented with $0,15 \mathrm{mM}$ ascorbic acid, $0.01 \mu \mathrm{M}$ dexamethasone, $10 \mathrm{mM}$ $\beta$-glycerolphosphate, $10 \mathrm{nM}$ cholecalciferol (OSTEO).

Multiple signalling pathways have been demonstrated to participate in the differentiation of an osteoblast progenitor to a committed osteoblast, including Transforming Growth Factor $\beta$ (TGF- $\beta$ )/BMP, Wnt/ $\beta$-catenin, Notch and Hedgehog. BMPs activate Runt-related protein 2 (Runx2) and Osterix (Osx), considered the master regulators for bone formation and the key transcriptional activators of osteogenic differentiation (Yoshimura et al. 2006). Binding sites for Runx 2 are present in genes whose products play a role in extracellular matrix mineralization, such as collagen type I and osteopontin (OPN), as well as in genes involved in cell growth and angiogenesis (Lian et al. 2006).

To assess the osteogenic differentiation of rbASCs, specific osteogenic proteins have been analyzed over time: alkaline phosphatase (ALP) and OPN for early osteogenesis, collagen type I (Coll I) and osteonectin (ONC) as intermediate phase markers, and osteocalcin $(\mathrm{OC})$ and bone mineralization for late osteogenesis.

rbASCs efficiently differentiate into osteoblast-like cells and a morphological change is observed already after $7 \mathrm{~d}$ of differentiation (Fig. 19.4A). ALP activity increases of about 168 percent compared to undifferentiated cells (Fig. 19.4B), and osteo-rbASCs show an induction of ONC at $7 \mathrm{~d}(+157.6$ percent), which is further up-regulated after $14 \mathrm{~d}$ (+1193.7 percent) (Fig. 19.4C). A similar pattern of expression is depicted for collagen type I (Fig. 19.4C). Indeed, densitometric analysis indicate a significant increase of 432.2 and 1233.9 percent after 7 and $14 \mathrm{~d}$, respectively (Fig. 19.4C).

These data are also confirmed by Sirius Red assay, which detects secreted collagen: this method is based on the ability of Sirius Red compound to selectively bind to fibrillar collagens (types I to V), specifically to the [Gly$X-Y]_{n}$ helical structure. Osteogenic differentiated cells produce significant amount of collagen, with an average increase of 105.9 percent respective to the same cells maintained in CTRL medium (Fig. 19.4D). Moreover, cells differentiated for $2 \mathrm{wk}$ produce calcified extracellular matrix (+161.6 percent versus undifferentiated rbASCs) (Fig. 19.4E). 
A

CTRL

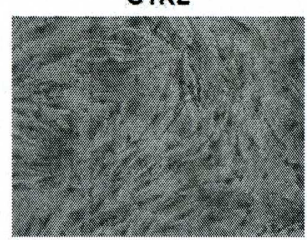

OSTEO

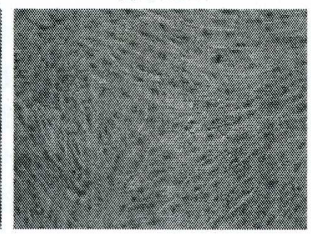

B

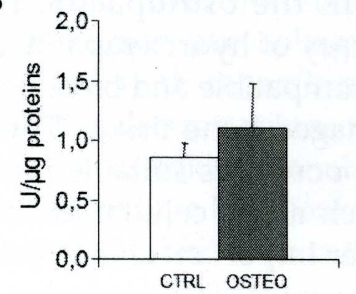

C
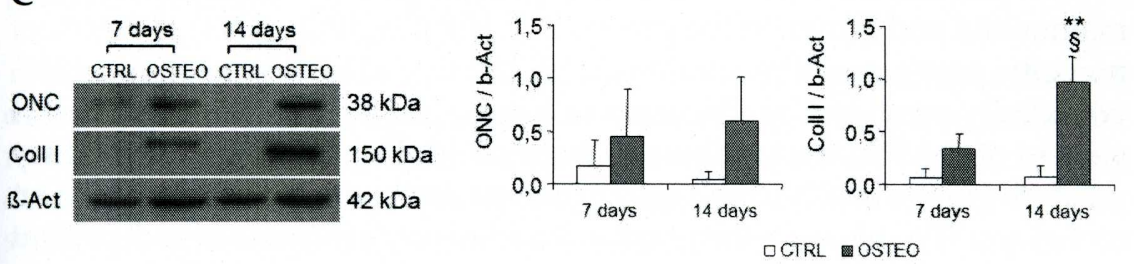

D

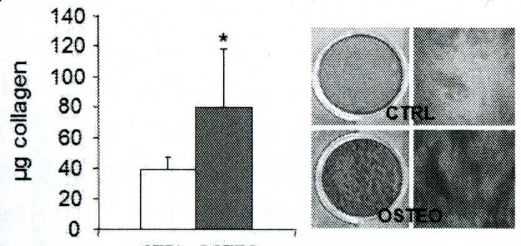

E

CTRL OSTEO

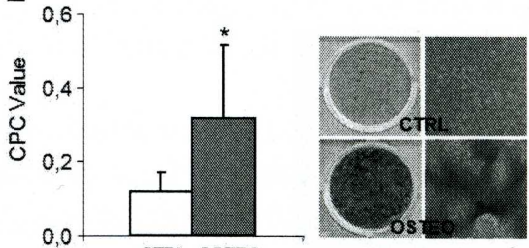

Figure 19.4 rbASCs differentiation towards osteoblasts-like cells. Morphological changes of rbASCs undifferentiated and differentiated for $7 \mathrm{~d}$ (A). ALP activity of rbASCs after $7 \mathrm{~d}$ of differentiation (mean $\pm \mathrm{SD}, \mathrm{n}=6$ ) (B). Osteonectin (ONC) and Coll I expression at 7 and $14 \mathrm{~d}$ in undifferentiated (CTRL) and osteogenic differentiated (OSTEO) rbASCs; western blot (left panel) and densitometric analysis (mean $\pm S D, n=2)$ (right panel) $(C)$. Collagen production by rbASCs cultured for $14 \mathrm{~d}$ in undifferentiated and osteogenic conditions (means $\pm S D, n=4$ ) and microphotographs of rbASCs maintained in CTRL or OSTEO medium stained with Sirius $\operatorname{Red}(D)$. Calcified extracellular matrix quantification (means $\pm S D, n=6$ ) produced by rbASCs cultured on polystyrene for $14 \mathrm{~d}$ in CTRL and OSTEO conditions (CPC, cetylpyridinium chloride extraction) and microphotographs of rbASCs stained with Alizarin Red-S (E). OSTEO vs CTRL: ${ }^{*} \mathrm{p}<.05 ;{ }^{* *} \mathrm{p}<.01 ; 14 \mathrm{~d}$ vs $7 \mathrm{~d}$ : $\$ \mathrm{p}<.05$ (modified by de Girolamo et al. 2011).

\section{In vitro rbASCs-hydroxyapatite Constructs}

Stem cells exist in tightly controlled niches and alteration in this microenvironment can modify their behaviour. Furthermore, stem cells are often involved in a disease or injury setting where inflammatory signals may be prevalent in recruiting them and in modulating their function. Biomaterials as scaffolds may potentially provide a controlled environment, and several studies have demonstrated the benefits of using mesenchymal stem cellscaffold constructs in regenerative medicine (Levi and Longaker 2011). 
In the orthopaedic field, various studies have established the efficacy of hydroxyapatite as a bone substitute. Hydroxyapatite (HA) is a biocompatible and bioactive material that can be used to restore or repair damaged bone tissue. This scaffold exhibits a strong bond to the bone, and possesses suitable mechanical properties and interconnecting pores which allow cellular infiltration, graft integration and vascularization. Other important characteristics of this scaffold are its osteoinductive and osteoconductive properties.

To confirm all these effects on rbASCs, undifferentiated cells are maintained and grown in the presence of $\mathrm{HA}\left(\mathrm{Ca}_{10}\left(\mathrm{PO}_{4}\right)_{6}(\mathrm{OH})_{2}, 70-80 \%\right.$ of porosity, pore size $<10 \mu \mathrm{m}, \approx 3 \%$ vol; $10-150 \mu \mathrm{m}, \approx 11 \%$ vol; $>150 \mu \mathrm{m}, \approx 86 \%$ vol, kindly provided by Finceramica S.p.A., Italy) (Fig. 19.5A). $97.1 \pm 2.4$ percent of rbASCs finely adhere to the scaffold (Fig. 19.5B and D) and they produce greater levels of collagen in comparison with cells maintained on monolayer (PA, plastic adherence), with an average increase of 48.2 percent (Fig. 19.5C).
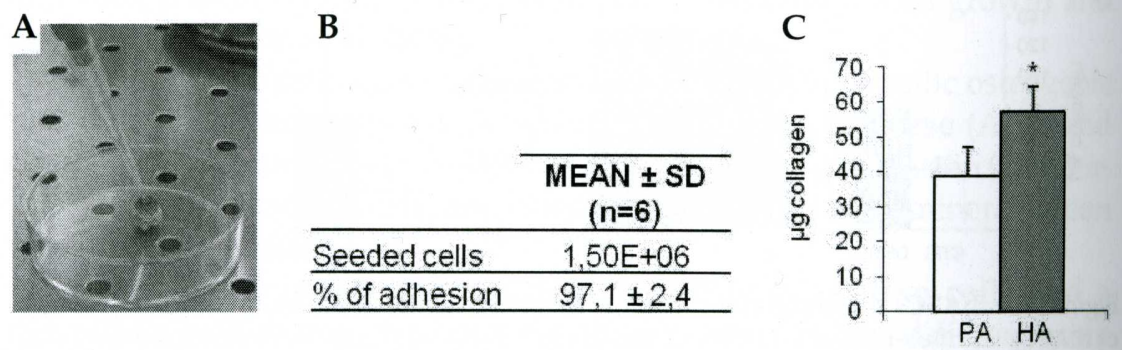

D

LIGHT MICROSCOPY

SEM ANALYSIS

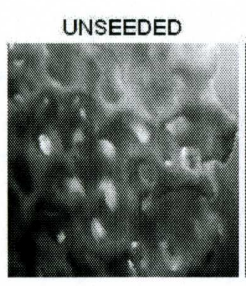

SEEDED
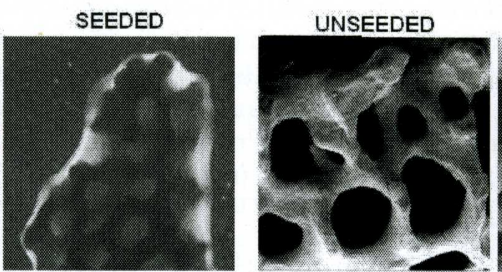

SEEDED

Figure 19.5 rbASCs-hydroxyapatite interaction. Loading of $1.5 \times 10^{6}$ undifferentiated rbASCs on hydroxyapatite disks (A). Percentages of adhesion of rbASCs in contact with the HA disk in vitro for $16 \mathrm{hr}$ (mean $\pm \mathrm{SD}, \mathrm{n}=6$ ) (modified by de Girolamo et al. 2011) (B). Quantification of collagen produced by undifferentiated rbASCs cultured on polystyrene (PA) or on hydroxyapatite (HA) for $14 \mathrm{~d}$ (means $\pm \mathrm{SD}, \mathrm{n}=4)(\mathrm{C})$. Light microscopy and SEM pictures of HA granules unseeded or seeded with rbASCs (with permission from Arrigoni et al. 2009) (D). HA vs PA: * $\mathrm{p}<.05$. 
Recently, Hao et al. (Hao et al. 2010a), fabricated a novel bioconstruct combining a nano-hydroxyapatite/recombinant human-like collagen/ poly(lactic acid) scaffold and rbASCs transfected with recombinant adenovirus vector producing human BMP-2. Scanning electron microscope (SEM) demonstrated integration of rbASCs within the scaffold and analysis of gene expression showed an up-regulation of collagen type I, osteonectin and osteopontin, indicating the osteogenic potential of rabbit stem cells.

\section{In vivo Models}

To adequately translate in vivo findings to the clinic, important pre-clinical data must be obtained in order to demonstrate the osteogenic ability of $\mathrm{rbASC}$ s to regenerate bone tissue. Many groups use either femoral or tibia defects as a bone load-bearing basis, seeding ASCs on osteoconductive scaffolds. Some reports demonstrated failed repair of bone defects in rat models by using ASCs incorporated in biomaterials (Li et al. 2007). In contrast, a more recent study by Hao et al. shows the use of rbASCs overexpressing BMP-2, to heal a femoral or tibial critical size bone defect (Hao et al. 2010a).

In addition, our group has also demonstrated that autologous and undifferentiated rbASCs seeded on clinical grade HA disks are a potential treatment for the regeneration of a critical size bone defect in rabbit tibia. The use of untreated rbASCs suggests the ability of these cells to achieve bone regeneration probably through the combined effect of the lesion's microenvironment and the scaffold. This evidence may be useful in a hypothetic one-step bone defect treatment, where mesenchymal stem cells could be purified from the donor tissue, directly seeded on scaffold and immediately implanted into the defect, obviating the necessity of either pre-stimulation or genetic modification (de Girolamo et al. 2011).

In this study, an $8 \mathrm{~mm}$ diameter full-thickness bone defect was created bilaterally in the proximal epiphysis of the medial facet of the rabbits' tibia (Fig. 19.6A). The choice to perform circular full-thickness defects instead of a periosteal segmental one was due to the fact that these kind of defects are more difficult to repair, and avoids the use of mechanical stabilization devices, which are known to affect bone healing, permitting to accurately evaluate the ability of autologous rbASCs to improve bone healing in a short follow-up of eight weeks.

HA scaffold constructs were implanted into the defect through a "press-fit" technique in group B and D (Fig. 19.6B, D and E), whereas, a semi-liquid suspension of rbASCs was directly injected in bone defects of group C (Fig. 19.6C and E). Group A was just untreated lesioned bones (Fig. 19.6A and E). 

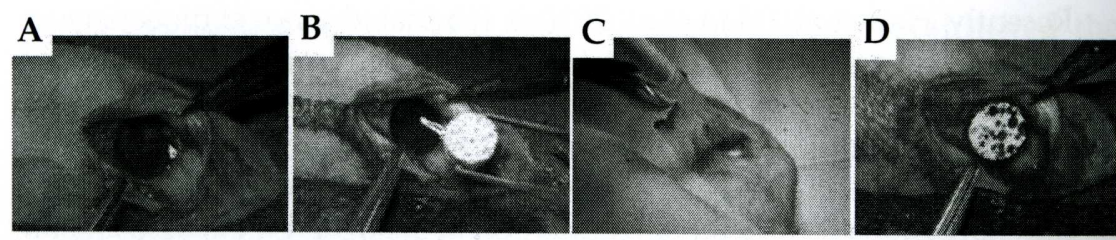

E

\begin{tabular}{cccc}
\hline Group & Defect Treatment & Side & $\mathbf{n}$ \\
\hline A & Untreated & Right & 6 \\
\hline B & HA & Left & 6 \\
\hline C & $1.5 \times 10^{6} \mathrm{rbASCs}$ & Right & 6 \\
\hline D & $1.5 \times 10^{6} \mathrm{rbASCs}+\mathrm{HA}$ & Left & 6 \\
\hline
\end{tabular}

Figure 19.6 Surgical procedure and constructs implantation. Critical size tibia defect (A), implantation of HA scaffold alone (B) or in association with rbASCs into the defect (D), and filling of the lesion site with a semi-liquid suspension of rbASCs (C). Experimental scheme of the bone defects treatment (E) (modified by de Girolamo et al. 2011).

\section{Gross Appearance Analyses and Radiological Assessment}

To evaluate the surgery outcome and the state of bone healing during the study, lateral radiographs were taken immediately after surgery $\left(T_{0}\right), 6 \mathrm{wk}$ after intervention $\left(\mathrm{T}_{6}\right)$ and post-tibia removal $\left(8 \mathrm{wk}, \mathrm{T}_{8}\right)$.

At $\mathrm{T}_{0}$, all the scaffolds were correctly placed and no bone fracture was seen at the lesion site (Fig. 19.7, $\mathrm{T}_{0}$ ). A second radiographic analysis was performed $6 \mathrm{wk}$ after implantation and no signs of osteolysis, fracture, or osteopenia were observed: instead, all lesions showed signs of quite an advanced bone remodelling process (Fig. 19.7, $\mathrm{T}_{6}$ ).

Eight weeks later, the animals were sacrificed, tibia explanted and the scoring system showed in Table 19.1a was used to evaluate bone integration in terms of good repair for the groups with or without scaffold or integration of the HA disks.

In all the groups, bone defects were satisfactorily filled and, according to the modified Wakitani scale, the stiffness was considered good (Wakitani et al. 1994). Indeed, no significant differences were observed between sham and rbASCs treated group ( $\mathrm{p}>.05$, Table 19.1b). In addition, in groups with implanted scaffold no significant differences have been observed between empty or rbASCs seeded ones ( $p>05$, Table 19.1b).

X-rays bone defects of group A seemed to be partially filled in a nonhomogeneous way, whereas the use of rbASCs only (group C) allowed a more complete and homogeneous filling of the lesion. In both groups 
$T_{0}$

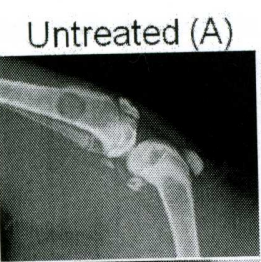

$\mathrm{T}_{6}$

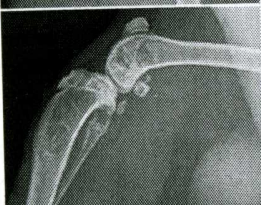

$H A(B)$

$\operatorname{rbASCs}(\mathrm{C}) \quad \mathrm{rbASCs}+\mathrm{HA}(\mathrm{D})$

$\mathrm{T}_{8}$
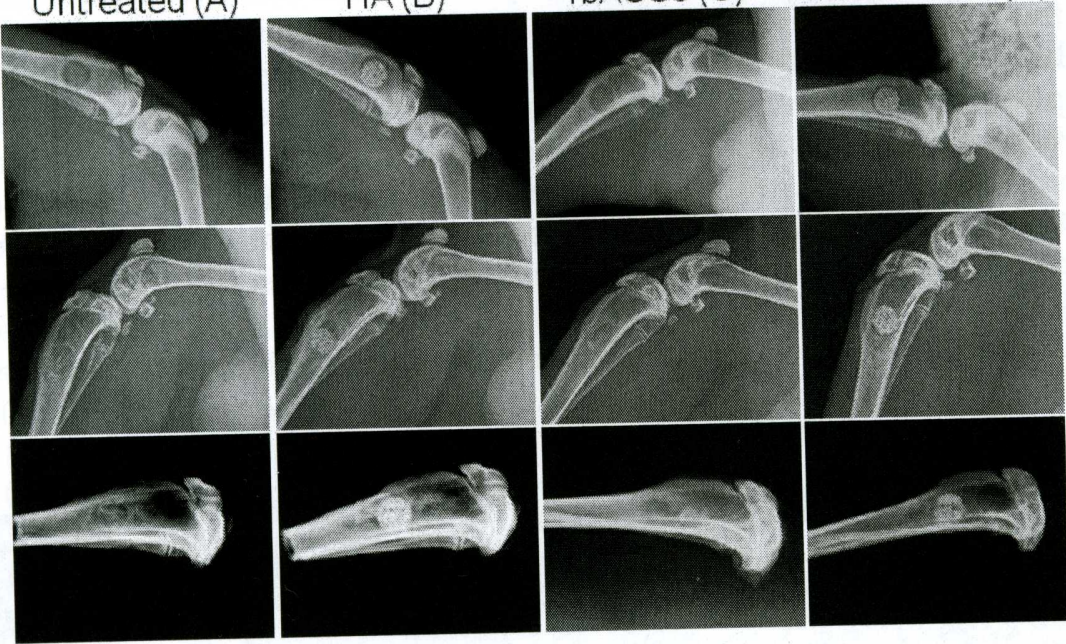

Figure 19.7 X-rays of lesioned bones. Rabbits were X-rayed immediately after surgical treatment $\left(T_{0}\right)$, after $6 \mathrm{wk}$ from intervention $\left(\mathrm{T}_{6}\right)$, and after tibia removal $\left(8 \mathrm{wk}, \mathrm{T}_{8}\right)$. Untreated group (group A), hydroxyapatite-alone treated group (group B), only rbASCs treated group (group C) and rbASCs-hydroxyapatite treated group (group D) (with permission from de Girolamo et al. 2011).

Table 19.1 Evaluation of the bone regeneration process by gross appearance analyses.

\begin{tabular}{|cc|ccc|}
\hline a & b & & \\
\hline \multicolumn{2}{|c|}{ Gross Appearance Analyses } & Group & \multirow{2}{*}{ Filling } & \multirow{2}{*}{ Stiffness } \\
& Stiffness & A & 2,83 & 2,83 \\
\hline Filling & Same stiffness $=3$ & B & 2,67 & 2,83 \\
\hline Same level $=3$ & Softer $=2$ & C & 2,50 & 2,50 \\
\hline Overgrowth $=2$ & Very soft $=1$ & D & 2,67 & 3,00 \\
\hline Undergrowth $>1 \mathrm{~mm}=1$ &
\end{tabular}

Filling and stiffness following modified Wakitani scoring scale (a) of the tibia explanted from the four animal groups A, untreated; $\mathrm{B},+\mathrm{HA} ; \mathrm{C},+\mathrm{rbASC} ; \mathrm{D},+\mathrm{rbASC}+\mathrm{HA}(\mathrm{b})$. (modified by de Girolamo et al. 2011).

$\mathrm{B}$ and $\mathrm{D}$, the scaffolds showed signs of good osteointegration, even if a conspicuous amount of HA was not reabsorbed and was still clearly evident (Fig. 19.7, $\mathrm{T}_{8}$ ).

\section{Histology and Immunohistochemistry}

Histology and immunohistochemistry analyses were performed with the aim of identifying the nature of new formed bone tissue. Lee et al. have recently reported that the use of different hydroxyapatite supports induces well-recovered cortical bone with newly developed regular bone matrix 
arranging, concluding that HA may be suitable as a bone substitute in the rabbit tibial defect model (Lee et al. 2010). However, the efficacy of bone substitutes could be ameliorated combining the osteoconductive properties of hydroxyapatite with the osteogenic potential of mesenchymal stem cells. As previously shown, rbASCs are able to colonize scaffolds and move along the interconnected pores, thus contributing to a better bone tissue regeneration. de Girolamo et al. reported that the use of rbASCs alone, administered as a semi-liquid suspension directly injected in the lesion site (group C) induces formation of new bone able to fill the defect, even if a woven matrix conformation indicates an immature bone tissue (Fig. 19.8) (de Girolamo et al. 2011).

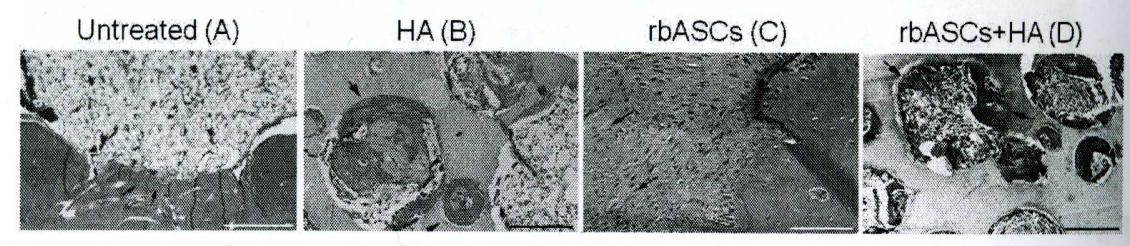

Figure 19.8 Histological analyses of sections from decalcified tibial samples. Haematoxylineosin staining of newly formed tissue at the lesion site of the four experimental groups. Untreated bone (A), plus scaffold (B), only cells (C), a bioconstruct rbASCs+HA (D). In group $B$ and D, the new-formed bone within the scaffold pores (arrows) shows a lamellar organization. The reparation tissue in the site of the defect of group $C$ shows a distinct woven matrix (arrows). (A, scale bar-500 $\mu \mathrm{m}$; B, scale bar-100 $\mu \mathrm{m} ; C, D$, scale bar-200 $\mu \mathrm{m}$ ) (with permission from de Girolamo et al. 2011).

The combination of rbASCs seeded on HA disks induces an abundant new bone formation. Pores are filled more homogeneously in comparison to the group treated with only $\mathrm{HA}$, and along the walls new mature bone is observed, as well as connective fibrous tissue in the inner part, indicating an ongoing bone maturation process. Osteoblasts are observed in conjunction with bone trabecules, located at the periphery of the scaffold pores which display a lamellar matrix (Fig. 19.8). These results are also confirmed by OPN and Coll I expressions which are observed in the HA treated bone, just in conjunction with bone trabecules located at the periphery of the scaffold pores, whereas, in rbASCs-HA implanted group, the specific expression is also detected in the middle of the pores (unpublished data). These data indicate that the bone maturation process is more advanced in the celltreated defects, where lamellar bone is largely represented.

\section{Biomechanical Test}

Defects treated with rbASCs show improved mechanical properties, tested by nanoindentation experiments using a NanoTest Indenter System (Micro Materials Ltd., UK) with a diamond Berkovich indenter tip. 
Nanoindentation is derived from the classical hardness test, but it is carried out on a much reduced scale. It can be used to determine the hardness of thin layers (non-decalcified samples-thickness $\sim 40 \mu \mathrm{m}$ ) as well as material properties such as elasticity (Er), hardness $(\mathrm{H})$, plasticity, and tensile strength. These measurements involve applying a small force $(1 \mathrm{mN}, 5 \mathrm{mN}$, and $50 \mathrm{mN})$ to a sample using a sharp probe and measuring the resultant penetration depth. The measured values are used to calculate the contact area and hence the particular properties of the sample material.

The rbASCs-HA constructs have an improved capability to bear mechanical loading. This is quite evident at low forces $(1 \mathrm{mN}$, corresponding to $200 \mathrm{~nm}$ of penetration depth), where it is possible to observe an increase in stiffness and in hardness in the rbASCs-treated group with respect to HA-group. This result might be explained by a higher mineral content present in the lesions treated with rbASCs-HA, thus representing a more advanced step in the healing process (unpublished data).

In conclusion, we suggest the use of ASCs as a safe cellular therapy in future clinical applications where a large bone defect needs to be treated. Indeed, the treatment of bone lesions with autologous rbASCshydroxyapatite bioconstructs have improved bone healing, and these results have paved the way for a new study on a large size animal model.

\section{Key Facts}

Table 19.2 Key features of ASCs and bone repair.

1. Critical-size bone defects may occur due to trauma, diseases or tumours.

2. The implant of a cells-scaffold tissue engineering construct represents a valid alternative to heterologous or autologous bone.

3. ASCs are optimum candidates in tissue engineering applications for their mesenchymal stem cells features together with their easily availability.

4. Biomaterials, such as hydroxyapatite, are able to osteo-induce ASCs allowing these cells to improve tissue repair.

5. Animal models are required before moving to the clinic.

6. The easy handling and bone similarities between humans and rabbits make this animal a good model in the orthopaedic field.

7. The rabbit tibia regenerated with autologous cells-hydroxyapatite construct leads to a mature tissue similar to the native one.

This table lists the key facts of tissue engineering applied to the regeneration of bone by autologous rabbit ASCs in association with hydroxyapatite. ASCs: Adipose-derived Stem Cells. 


\section{Dictionary of Key Terms, Genes, Chemicals or Pathways}

- Adipose-derived stem cells: mesenchymal stem cells isolated from subcutaneous or omental adipose tissue.

- Bioconstructs: combination of progenitor or terminally differentiated cells with either natural or synthetic scaffolds able or not to release active molecules, and to adapt to the wounded tissue.

- Critical-size bone defect: the smallest size intra-osseous wound in a particular bone that will not spontaneously heal during the life span.

- Pre-clinical model: designed experimental study with the minimum number of animals to determine treatment toxicity and therapeutic effects, and which requires ethical approval.

- Immunohistochemistry: technique based on the binding of specific antibodies to cellular antigens used to evaluate the distribution and localization of specific biomarkers in fixed tissues.

- Nanoindentation: technique which exploits diamond-coated indenters to measure mechanical properties such as hardness and elasticity of several tissues.

\section{Summary Points}

- Fat represents an ideal source of mesenchymal stem cells due to the high number of cells which can be isolated through simple procedures (both surgical and enzymatic). Adipose-derived stem cells (ASCs) are able to self-renew in culture for a long time, are multipotent and low immunogenic, and possess immunomodulatory and anti-inflammatory features.

- ASCs can be associated with scaffolds in tissue engineering approaches.

- Preclinical studies are always required before moving to the clinical phase.

- The rabbit is a good animal model in the orthopaedic field, and rabbit ASCs (rbASCs) can be easily isolated from the interscapular region of New Zealand White rabbits.

- rbASCs rapidly proliferate and show clonogenic ability and osteogenic potential in vitro.

- Hydroxyapatite (HA) is largely used in clinic for its osteoinductive property; in vitro experiments with rbASCs confirm this material's feature.

- rbASCs-HA bioconstructs have been used to repair a critical size tibia defect. 
- X-rays, histology and immunohistochemistry analyses revealed a good quality of the neo-formed bone tissue.

- Nanoindentation tests confirm improved bone elasticity and hardness.

\section{Acknowledgment}

The authors sincerely thank Drs. L. de Girolamo, A. Addis, M. Campagnol, C. Domeneghini, A. Di Giancamillo, P. Stortini and D. Carnelli for their valuable work and Finceramica S.p.A. (Faenza, Italy) for providing the scaffolds. This work has been partially supported by the Italian Ministry of University and Research (2006091907_003) and the Italian Ministry of Health (2007-656853).

\section{List of Abbreviations}

$\begin{array}{lll}\text { ALP } & : & \text { alkaline phosphatase } \\ \text { ASCs } & : & \text { adipose-derived stem cells } \\ \text { BMD } & : & \text { bone mineral density } \\ \text { BMP } & : & \text { bone morphogenic proteins } \\ \text { BMSCs } & : & \text { bone marrow mesenchymal stem cells } \\ \text { CFU-F } & : & \text { colony forming unit-fibroblast } \\ \text { CFU-O } & : & \text { colony forming unit-osteoblast } \\ \text { Coll I } & : & \text { collagen type I } \\ \text { CPC } & : & \text { cetylpyridinium chloride } \\ \text { CTRL } & : & \text { undifferentiative medium } \\ \text { DMEM } & : & \text { Dulbecco's modified eagle's medium } \\ \text { EDTA } & : & \text { ethylenediaminetetraacetic acid } \\ \text { Er } & : & \text { elasticity } \\ \text { FBS } & : & \text { fetal bovine serum } \\ \text { H } & : & \text { hardness } \\ \text { HA } & : & \text { hydroxyapatite } \\ \text { MHC } & : & \text { major histocompatibility complex } \\ \text { MSCs } & : & \text { mesenchymal stem cells } \\ \text { MTT } & : & \text { (3-(4, 5-dimethylthiazolyl-2)-2, 5-diphenyltetrazolium } \\ & & \text { bromide } \\ \text { OC } & : & \text { osteocalcin } \\ \text { ONC } & : & \text { osteonectin } \\ \text { OPN } & : & \text { osteopontin } \\ \text { OSTEO } & : & \text { osteogenic medium } \\ \text { OSX } & : & \text { osterix } \\ \text { PA } & : & \text { plastic adherence } \\ & & \end{array}$




$\begin{array}{lll}\text { PBS } & : & \text { phosphate-buffered saline } \\ \text { PLGA } & : & \text { poly(lactic-co-glycolic acid) } \\ \text { Runx2 } & : & \text { Runt-related protein } 2 \\ \text { SEM } & : & \text { scanning electron microscope } \\ \text { SVF } & : & \text { stromal vascular fraction } \\ \beta \text {-TCP } & : & \beta \text {-tricalcium phosphate } \\ \text { TGF- } \beta & : & \text { transforming growth factor } \beta\end{array}$

\section{References}

Albrektsson, T. and C. Johansson. 2001. Osteoinduction, osteoconduction and osseointegration. Eur Spine J. 10 Suppl. 2. S96-101.

Arrigoni, E., S. Lopa, L. de Girolamo, D. Stanco and A.T. Brini. 2009. Isolation, characterization and osteogenic differentiation of adipose-derived stem cells: from small to large animal models. Cell Tissue Res. 338: 401-411.

Castaneda, S., R. Largo, E. Calvo, F. Rodriguez-Salvanes, M.E. Marcos, M. Diaz-Curiel and G. Herrero-Beaumont. 2006. Bone mineral measurements of subchondral and trabecular bone in healthy and osteoporotic rabbits. Skeletal Radiol. 35: 34-41.

Choi, E.W., I.S. Shin, S.Y. Park, J.H. Park, J.S. Kim, E.J. Yoon, S.K. Kang, J.C. Ra and S.H. Hong. 2012. Reversal of serological, immunological and histological dysfunction in systemic lupus erythematosus mice by long-term serial adipose tissue-derived mesenchymal stem cell transplantation. Arthritis Rheum. 64: 243-253.

Chu, C.R., M. Szczodry and S. Bruno. 2010. Animal models for cartilage regeneration and repair. Tissue Eng Part B Rev. 16: 105-15.

Constantin, G., S. Marconi, B. Rossi, S. Angiari, L. Calderan, E. Anghileri, B. Gini, S.D. Bach, M. Martinello, F. Bifari, M. Galie, E. Turano, S. Budui, A. Sbarbati, M. Krampera and B. Bonetti. 2009. Adipose-derived mesenchymal stem cells ameliorate chronic experimental autoimmune encephalomyelitis. Stem Cells. 27: 2624-2635.

Dashtdar, H., H.A. Rothan, T. Tay, R.E. Ahmad, R. Ali, L.X. Tay, P.P. Chong and T. Kamarul. 2011. A preliminary study comparing the use of allogenic chondrogenic pre-differentiated and undifferentiated mesenchymal stem cells for the repair of full thickness articular cartilage defects in rabbits. J Orthop Res. 29: 1336-1342.

de Girolamo, L., M.F. Sartori, E. Arrigoni, L. Rimondini, W. Albisetti, R.L. Weinstein and A.T. Brini. 2008. Human adipose-derived stem cells as future tools in tissue regeneration: osteogenic differentiation and cell-scaffold interaction. Int J Artif Organs. 31: 467-479.

de Girolamo, L., E. Arrigoni, D. Stanco, S. Lopa, A. Di Giancamillo, A. Addis, S. Borgonovo, C. Dellavia, C. Domeneghini and A.T. Brini. 2011. Role of autologous rabbit adiposederived stem cells in the early phases of the repairing process of critical bone defects. J Orthop Res. 29: 100-108.

Di Nicola, M., C. Carlo-Stella, M. Magni, M. Milanesi, P.D. Longoni, P. Matteucci, S. Grisanti and A.M. Gianni. 2002. Human bone marrow stromal cells suppress T-lymphocyte proliferation induced by cellular or nonspecific mitogenic stimuli. Blood. 99: 3838-3843.

Dudas, J.R., K.G. Marra, G.M. Cooper, V.M. Penascino, M.P. Mooney, S. Jiang, J.P. Rubin and J.E. Losee. 2006. The osteogenic potential of adipose-derived stem cells for the repair of rabbit calvarial defects. Ann Plast Surg. 56: 543-548.

Friedenstein, A.J., U.F. Deriglasova, N.N. Kulagina, A.F. Panasuk, S.F. Rudakowa, E.A. Luria and I.A. Ruadkow. 1974. Precursors for fibroblasts in different populations of hematopoietic cells as detected by the in vitro colony assay method. Exp Hematol. 2: 83-92.

Gilsanz, V., T.F. Roe, D.T. Gibbens, E.E. Schulz, M.E. Carlson, O. Gonzalez and M.I. Boechat. 1988. Effect of sex steroids on peak bone density of growing rabbits. Am J Physiol. 255: E416-421. 
Gonzalez, M.A., E. Gonzalez-Rey, L. Rico, D. Buscher and M. Delgado. 2009. Treatment of experimental arthritis by inducing immune tolerance with human adipose-derived mesenchymal stem cells. Arthritis Rheum. 60: 1006-1019.

Hao, W., J. Dong, M. Jiang, J. Wu, F. Cui and D. Zhou. 2010a. Enhanced bone formation in large segmental radial defects by combining adipose-derived stem cells expressing bone morphogenetic protein 2 with nHA/RHLC/PLA scaffold. Int Orthop. 34: 1341-1349.

Hao, W., L. Pang, M. Jiang, R. Lv, Z. Xiong and Y.Y. Hu. 2010b. Skeletal repair in rabbits using a novel biomimetic composite based on adipose-derived stem cells encapsulated in collagen I gel with PLGA-beta-TCP scaffold. J Orthop Res. 28: 252-257.

Im, G.I. and J.H. Lee. 2010. Repair of osteochondral defects with adipose stem cells and a dual growth factor-releasing scaffold in rabbits. J Biomed Mater Res B Appl Biomater. 92: 552-560.

Kern, S., H. Eichler, J. Stoeve, H. Kluter and K. Bieback. 2006. Comparative analysis of mesenchymal stem cells from bone marrow, umbilical cord blood, or adipose tissue. Stem Cells. 24: 1294-1301.

Lee, M.J., S.K. Sohn, K.T. Kim, C.H. Kim, H.B. Ahn, M.S. Rho, M.H. Jeong and S.K. Sun. 2010. Effect of hydroxyapatite on bone integration in a rabbit tibial defect model. Clin Orthop Surg. 2: 90-97.

Levi, B. and M.T. Longaker. 2011. Concise review: adipose-derived stromal cells for skeletal regenerative medicine. Stem Cells. 29: 576-582.

Li, H., K. Dai, T. Tang, X. Zhang, M. Yan and J. Lou. 2007. Bone regeneration by implantation of adipose-derived stromal cells expressing BMP-2. Biochem Biophys Res Commun. 356: 836-842.

Li, J., Y. Li, S. Ma, Y. Gao, Y. Zuo and J. Hu. 2010. Enhancement of bone formation by BMP-7 transduced MSCs on biomimetic nano-hydroxyapatite/polyamide composite scaffolds in repair of mandibular defects. J Biomed Mater Res A. 95: 973-981.

Li, W.J., H. Chiang, T.F. Kuo, H.S. Lee, C.C. Jiang and R.S. Tuan. 2009. Evaluation of articular cartilage repair using biodegradable nanofibrous scaffolds in a swine model: a pilot study. J Tissue Eng Regen Med. 3: 1-10.

Lian, J.B., G.S. Stein, A. Javed, A.J. Van Wijnen, J.L. Stein, M. Montecino, M.Q. Hassan, T. Gaur, C.J. Lengner and D.W. Young. 2006. Networks and hubs for the transcriptional control of osteoblastogenesis. Rev Endocr Metab Disord. 7: 1-16.

Lovett, M., K. Lee, A. Edwards and D.L. Kaplan. 2009. Vascularization strategies for tissue engineering. Tissue Eng Part B Rev. 15: 353-370.

Najar, M., G. Raicevic, H.I. Boufker, H. Fayyad-Kazan, C. De Bruyn, N. Meuleman, D. Bron, M. Toungouz and L. Lagneaux. 2010. Adipose-tissue-derived and Wharton's jelly-derived mesenchymal stromal cells suppress lymphocyte responses by secreting leukemia inhibitory factor. Tissue Eng Part A. 16: 3537-3546.

Neyt, J.G., J.A. and N.C. Carroll. 1998. Use of animal models in musculoskeletal research. Iowa Orthop J. 18: 118-123.

Oshima, S., M. Ishikawa, Y. Mochizuki, T. Kobayashi, Y. Yasunaga and M. Ochi. 2010. Enhancement of bone formation in an experimental bony defect using ferumoxidelabelled mesenchymal stromal cells and a magnetic targeting system. J Bone Joint Surg Br. 92: 1606-1613.

Pieri, F., E. Lucarelli, G. Corinaldesi, N.N. Aldini, M. Fini, A. Parrilli, B. Dozza, D. Donati and C. Marchetti. 2010. Dose-dependent effect of adipose-derived adult stem cells on vertical bone regeneration in rabbit calvarium. Biomaterials. 31: 3527-3535.

Sarkar, M.R., P. Augat, S.J. Shefelbine, S. Schorlemmer, M. Huber-Lang, L. Claes, L. Kinzl and A. Ignatius. 2005. Bone formation in a long bone defect model using a platelet-rich plasma-loaded collagen scaffold. Biomaterials. 27: 1817-23.

Schaffler, A. and C. Buchler. 2007. Concise review: adipose tissue-derived stromal cells-basic and clinical implications for novel cell-based therapies. Stem Cells. 25: 818-827.

Spencer, N.D., J.M. Gimble and M.J. Lopez. 2011. Mesenchymal stromal cells: past, present, and future. Vet Surg. 40: 129-139. 
Torres, F.C., C.J. Rodrigues, I.N. Stocchero and M.C. Ferreira. 2007. Stem cells from the fat tissue of rabbits: an easy-to-find experimental source. Aesthetic Plast Surg. 31: 574-578.

Torroni, A. 2009. Engineered bone grafts and bone flaps for maxillofacial defects: state of the art. J Oral Maxillofac Surg. 67: 1121-1127.

Wakitani, S., T. Goto, S.J. Pineda, R.G. Young, J.M. Mansour, A.I. Caplan and V.M. Goldberg. 1994. Mesenchymal cell-based repair of large, full-thickness defects of articular cartilage. J Bone Joint Surg Am. 76: 579-592.

Wang, L., H. Fan, Z.Y. Zhang, A.J. Lou, G.X. Pei, S. Jiang, T.W. Mu, J.J. Qin, S.Y. Chen and D. Jin. 2010. Osteogenesis and angiogenesis of tissue-engineered bone constructed by prevascularized beta-tricalcium phosphate scaffold and mesenchymal stem cells. Biomaterials. 31: 9452-9461.

Wang, X., J.D. Mabrey and C.M. Agrawal. 1998. An interspecies comparison of bone fracture properties. Biomed Mater Eng. 8: 1-9.

Yanez, R., M.L. Lamana, J. Garcia-Castro, I. Colmenero, M. Ramirez and J.A. Bueren. 2006. Adipose tissue-derived mesenchymal stem cells have in vivo immunosuppressive properties applicable for the control of the graft-versus-host disease. Stem Cells. 24: 2582-2591.

Yoshimura, K., T. Shigeura, D. Matsumoto, T. Sato, Y. Takaki, E. Aiba-Kojima, K. Sato, K. Inoue, T. Nagase, I. Koshima and K. Gonda. 2006. Characterization of freshly isolated and cultured cells derived from the fatty and fluid portions of liposuction aspirates. J Cell Physiol. 208: 64-76.

Zhao, M., J. Zhou, X. Li, T. Fang, W. Dai, W. Yin and J. Dong. 2011. Repair of bone defect with vascularized tissue engineered bone graft seeded with mesenchymal stem cells in rabbits. Microsurgery. 31: 130-137.

Zuk, P.A., M. Zhu, P. Ashjian, D.A. De Ugarte, J.I. Huang, H. Mizuno, Z.C. Alfonso, J.K. Fraser, P. Benhaim and M.H. Hedrick. 2002. Human adipose tissue is a source of multipotent stem cells. Mol Biol Cell. 13: 4279-4295. 